\title{
Orbit Design for General Relativity Experiments: Heliocentric and Mercury-centric Cases
}

\author{
Ryan S. Park *, Daniel J. Scheeres ${ }^{\dagger}$, Giacomo Giampieri国, \\ James M. Longuski $\stackrel{\S}{\$}$, and Ephraim Fischbach $\mathbb{\Phi}$
}

\begin{abstract}
Tracking a spacecraft near the Sun provides unique opportunities to estimate gravity parameters and to test fundamental theories of physics. We develop a detailed covariance analysis of such trajectories, analyzing the uncertainty distribution of the first order Parameterized PostNewtonian parameters $\left(\gamma\right.$ and $\beta$ ) and the solar quadrupole $\left(J_{2}\right)$ moment based on radiometric measurements. Our analysis includes the effect of station-location uncertainties and stochastic accelerations acting on the spacecraft. We present realistic estimates of these parameters considering both heliocentric and Mercury-centric trajectories.
\end{abstract}

\section{Introduction}

The theory of General Relativity (GR) can be tested by precisely measuring small changes in a spacecraft trajectory. Two possible trajectory design options for testing GR in a sub-light speed regime are: 1) a trajectory originating near the Sun; and 2) an orbit about the planet Mercury. The novel feature of these trajectories is the possibility of estimating the Parameterized Post-Newtonian (PPN) parameters $(\gamma$ and $\beta)$ and the solar quadrupole moment $\left(J_{2}\right)$ separately, since previous GR experiments generally cannot clearly disentangle $\gamma, \beta$, and $J_{2}$ from each other [1]. Precisely knowing the parameters $\gamma, \beta$, and $J_{2}$ allows us to determine how well GR describes the physical world.

One may consider the tests we are investigating as an extension of Mercury's perihelion shift or the deflection of light by the Sun. However, tracking spacecraft trajectories near the Sun is fundamentally different from these previous GR tests. The precession of Mercury's perihelion was based on the secular effect of GR over long timespans. In actuality, the transient oscillations of Mercury's orbit due to GR are much larger (over one orbit) than the secular effect; however, their amplitudes are small in absolute terms, and their effect averages out over time. Hence, only the secular effects can be measured over very long time baselines, and these provide estimates of linear combinations of $\gamma$ and $\beta$, such as the perihelion advance effect $(2+2 \gamma-\beta)$ or the Nordtvedt effect $(\eta=4 \beta-\gamma-3)$. In contrast, the transient deviations in the trajectory due to GR over a short period of time allow us to discriminate between the gravity parameters. Moreover, light bending experiments can only provide very accurate estimates of the parameter $\gamma$ from the calibration of the radiometric data [2] $\left[\gamma=1+(2.1 \pm 2.3) \times 10^{-5}\right]$; however, the parameters $\beta$ and $J_{2}$ are unobservable with these tests.

A potential sub-light GR test has been proposed by Longuski et al. 3. (2001) where they place the spacecraft on a heliocentric hyperbolic trajectory with perihelion at four solar radii. Later studies [4, 5] investigated how well the gravity parameters could be measured from this test via a detailed covariance analysis. Considering foreseeable improvements in tracking capability and dragfree technology, that analysis gives unbiased estimates of $\sigma_{\gamma}=8.90 \times 10^{-5}$ and $\sigma_{\beta}=4.09 \times 10^{-4}$. This paper revisits those earlier studies and considers the effect of different spacecraft orbit orientations has on our predicted uncertainties of $\gamma, \beta$, and $J_{2}$.

Tracking the orbit of a spacecraft in orbit about Mercury provides another design option for testing GR. The idea is similar to the heliocentric case; however, there is a critical difference in

\footnotetext{
* Graduate Research Assistant, Department of Aerospace Engineering, The University of Michigan, Ann Arbor, Michigan, sanghp@umich.edu

${ }^{\dagger}$ Associate Professor, Department of Aerospace Engineering, The University of Michigan, Ann Arbor, Michigan, Associate Fellow AIAA, Member AAS, scheeres@umich.edu

${ }^{\ddagger}$ Research Associate, Department of Mathematics, The Blackett Laboratory, Imperial College, London, UK, g.giampieri@ic.ac.uk

$\S$ Professor, School of Aeronautics and Astronautics, Purdue University, West Lafayette, Indiana, Associate Fellow AIAA, Member AAS, longuski@ecn.purdue.edu

๑Professor, Physics Department, Purdue University, West Lafayette, Indiana, Fellow APS, ephraim@physics.purdue.edu
} 
this experiment. The effect of GR on Mercury is much smaller than for the heliocentric case, and hence, one may expect poor estimates of the gravity parameters by tracking a Mercury orbiter. The significant distinctions from the heliocentric and Mercury-centric cases are that the stochastic perturbations acting on Mercury are much less than those acting on the spacecraft. Of course, a Mercury orbiter will itself be under influence of large stochastic perturbations, but we are using the spacecraft to measure Mercury's state to extract the gravity information. In other words, one may view this case as a spacecraft heliocentric orbit (i.e., a spacecraft following Mercury's trajectory) with very small stochastic perturbations and accurate a priori information.

\section{Overview of the Covariance Analysis}

\section{Initial State Covariance Analysis}

Our filter model is based on ordinary first-order least-squares approximation theory and is carried out using modern orbit determination procedures [6, 7]. The state vector we estimate in our analysis can be represented as $\mathbf{y}=\left[\begin{array}{lll}\mathbf{x}^{T} & \mathbf{p}^{T} & \mathbf{q}^{T}\end{array}\right]^{T}$, where $\mathbf{x}$ is the dynamics vector $(T$ represents transpose), $\mathbf{p}$ is the parameter vector (e.g., $\gamma$ and $\beta$ ), and $\mathbf{q}$ is the measurement model parameter vector (e.g., station-location vector). The dimensions of $\mathbf{x}, \mathbf{p}$, and $\mathbf{q}$ are $n, m$, and $l$, respectively. If we consider $\mathbf{y}$ as a Gaussian random vector with mean (nominal) vector $(\overline{\mathbf{y}})$ and covariance matrix $(\mathbf{P})$, the probability density function $\left(\mathbf{f}_{\mathbf{Y}}\right)$ and the probability of $\mathbf{y}$ lying in a region $\boldsymbol{\Gamma}$ can be defined as:

$$
\begin{aligned}
\mathbf{f}_{\mathbf{Y}}(\mathbf{y}) & =\frac{e^{-\frac{1}{2}(\mathbf{y}-\overline{\mathbf{y}})^{T} \mathbf{P}^{-1}(\mathbf{y}-\overline{\mathbf{y}})}}{(2 \pi)^{(n+m+l) / 2 \sqrt{\operatorname{det} \mathbf{P}}}} \\
\mathbf{P r}(\mathbf{y} \in \boldsymbol{\Gamma}) & =\int_{\boldsymbol{\Gamma}} \mathbf{f}_{\mathbf{Y}}\left(\mathbf{y}^{\prime}\right) d \mathbf{y}^{\prime}
\end{aligned}
$$

Hence, studying the covariance matrix essentially provides us with the behavior of the uncertainties in the state $\mathbf{y}$. Moreover, if we let $\mathbf{P}_{o}$ be the initial covariance matrix, the covariance matrix at $t_{k}$ can be obtained by computing,

$$
\mathbf{P}_{k}=\boldsymbol{\Phi}\left(t_{k}, t_{o}\right) \mathbf{P}_{o} \boldsymbol{\Phi}^{T}\left(t_{k}, t_{o}\right)
$$

where $\mathbf{\Phi}\left(t_{k}, t_{o}\right)$ is the state transition matrix (STM) which maps the state perturbations from $t_{o}$ to $t_{k}$ as a function of time and is defined by the initial spacecraft state vector [i.e., $\boldsymbol{\Phi}\left(t_{k}, t_{o} ; \mathbf{r}_{o}, \mathbf{v}_{o}, \gamma, \beta\right)$ ]. Under ideal conditions (i.e., no stochastic perturbation), studying $\mathbf{P}_{k}$ or $\mathbf{P}_{o}$ gives essentially the same uncertainty information for the parameters (i.e., $\gamma, \beta$, and $J_{2}$ ). For the given state vector, the time propagated STM can represented as,

$$
\boldsymbol{\Phi}\left(t_{k}, t_{o}\right)=\frac{\partial \mathbf{y}\left(t_{k}\right)}{\partial \mathbf{y}\left(t_{o}\right)},
$$

with $\boldsymbol{\Phi}\left(t_{\mathrm{o}}, t_{\mathrm{o}}\right)$ an identity matrix. The time derivative of the STM is a simple linear relation, $\dot{\mathbf{\Phi}}\left(t_{k}, t_{o}\right)=\mathbf{A} \mathbf{\Phi}\left(t_{k}, t_{o}\right)$, where the linear mapping matrix $\mathbf{A}$ is given by,

$$
\mathbf{A}(t)=\frac{\partial}{\partial \mathbf{y}}\left(\frac{d \mathbf{y}}{d t}\right)
$$

Numerical precision is often lost in the computation of covariance due to numerical integration and matrix inversion error. A method for retaining precision is to use the Square Root Information Filter (SRIF) 7]:

$$
\mathbf{\Lambda}\left(t_{o} ; t_{k}, t_{o}\right)=\mathbf{R}^{T}\left(t_{o} ; t_{k}, t_{o}\right) \mathbf{R}\left(t_{o} ; t_{k}, t_{o}\right)
$$

Here $\boldsymbol{\Lambda}\left(t_{\mathrm{o}} ; t_{\mathrm{o}}, t_{k}\right)$ is the initial state information matrix in the interval from $t_{o}$ to $t_{k}$, and $\mathbf{R}\left(t_{o} ; t_{k}, t_{o}\right)$ is the initial state SRIF matrix which we propagate, instead of the information matrix, to update data in each time increment. The SRIF matrix is related to the adjoint of the state transition matrix (STM) and ideally maps as

$$
\mathbf{R}\left(t_{k} ; t_{k}, t_{o}\right)=\mathbf{R}\left(t_{o} ; t_{k}, t_{o}\right) \mathbf{\Phi}\left(t_{o}, t_{k}\right)
$$


Let $\mathbf{T}_{H}$ be an orthogonal Householder transformation matrix such that the updated SRIF matrix is defined as

$$
\mathbf{R}\left(t_{o} ; t_{k+1}, t_{o}\right)=\mathbf{T}_{H}\left[\begin{array}{c}
\mathbf{R}\left(t_{o} ; t_{k}, t_{o}\right) \\
\boldsymbol{\Sigma}^{-1} \mathbf{H}_{t_{k+1}} \mathbf{\Phi}\left(t_{k+1}, t_{o}\right)
\end{array}\right]=\left[\begin{array}{c}
\mathbf{R}_{H}\left(t_{o} ; t_{k+1}, t_{o}\right) \\
0
\end{array}\right],
$$

where $\boldsymbol{\Sigma}$ is the measurement noise matrix with corresponding values from Table $1, \mathbf{H}_{k+1}$ is the measurement partial matrix computed at $t_{k}$, and $\mathbf{R}_{H}$ is an upper triangular matrix. In the computation, we adopt QR-factorization as the Householder transformation [7]. After the Householder transformation is applied, the updated information matrix becomes

$$
\boldsymbol{\Lambda}\left(t_{o} ; t_{k+1}, t_{o}\right)=\mathbf{R}_{H}^{T}\left(t_{o} ; t_{k+1}, t_{o}\right) \mathbf{R}_{H}\left(t_{o} ; t_{k+1}, t_{o}\right),
$$

and the updated covariance matrix is

$$
\mathbf{P}\left(t_{o} ; t_{k+1}, t_{o}\right)=\boldsymbol{\Lambda}^{-1}\left(t_{o} ; t_{k+1}, t_{o}\right)=\mathbf{R}_{H}^{-1}\left(t_{o} ; t_{k+1}, t_{o}\right) \mathbf{R}_{H}^{-T}\left(t_{o} ; t_{k+1}, t_{o}\right),
$$

which represents the evolution of the a priori uncertainties in the state vector.

Table 1 Summary of Measurement Accuracies

\begin{tabular}{cccc}
\hline \hline $\begin{array}{c}\text { Tracking } \\
\text { System }\end{array}$ & $\sigma_{R}{ }^{\mathrm{a}}(\mathrm{m})$ & $\sigma_{V^{\mathrm{b}}}(\mathrm{nrad})$ & $\begin{array}{c}\sigma_{D}^{\mathrm{c}}(\mathrm{mm} / \mathrm{s}) \\
\text { (for } 1 \text { minute count time) }\end{array}$ \\
\hline X-band & 1 & 1 & 0.1 \\
K-band & 0.1 & 0.1 & 0.01 \\
\hline \hline
\end{tabular}

a. Range measurement accuracy.

b. VLBI measurement accuracy.

c. Doppler measurement accuracy.

\section{Current State Covariance Analysis}

The perturbations due to process noise (stochastic accelerations) can most easily be included in the current state covariance analysis $[\underline{8}$. Therefore, we directly solve for the current state SRIF matrix to include time-correlated random accelerations. We define the current-state information matrix and related quantities as

$$
\begin{aligned}
\boldsymbol{\Lambda}\left(t_{k} ; t_{k}, t_{o}\right) & =\mathbf{R}^{T}\left(t_{k} ; t_{k}, t_{o}\right) \mathbf{R}\left(t_{k} ; t_{k}, t_{o}\right), \\
\mathbf{P}\left(t_{k} ; t_{k}, t_{o}\right) & =\boldsymbol{\Lambda}^{-1}\left(t_{k} ; t_{k}, t_{o}\right), \\
\dot{\mathbf{R}}\left(t_{k} ; t_{k}, t_{o}\right) & =-\mathbf{R}\left(t_{k} ; t_{k}, t_{o}\right) \mathbf{A}\left(t_{k}\right) .
\end{aligned}
$$

The current state covariance matrix (Eq. 12) represents the uncertainties in the state variables at current time $t_{k}$, whereas the previous definition was for the a priori estimates. Again, the resulting $\gamma, \beta$, and $J_{2}$ uncertainties do not change since they are not dynamical variables. In the absence of stochastic perturbations, the two definitions of covariance matrices can be mapped into each other by Eq. (3); however, this ideal relation is no longer preserved in the presence of stochastic perturbations. The time derivative of the perturbed SRIF matrix is [8],

$$
\dot{\mathbf{R}}\left(t_{k} ; t_{k}, t_{o}\right)=-\mathbf{R}\left(t_{k} ; t_{k}, t_{o}\right) \mathbf{A}\left(t_{k}\right)-\frac{1}{2} \mathbf{R}\left(t_{k} ; t_{k}, t_{o}\right) \mathbf{B} \mathbf{P}_{\omega} \mathbf{B}^{T} \mathbf{R}^{T}\left(t_{k} ; t_{k}, t_{o}\right) \mathbf{R}\left(t_{k} ; t_{k}, t_{o}\right),
$$

where

$$
\mathbf{P}_{\omega}=2 \tau \sigma_{a}^{2} \mathbf{I}_{3 \times 3} .
$$

Here, $\mathbf{B}$ is a matrix mapping the process noise into the spacecraft velocity space, $\tau$ is the correlation time of the process noise, $\sigma_{a}$ is the steady-state stochastic acceleration, and the linear map $\mathbf{A}$ is defined as earlier. We assume that the stochastic accelerations are not being estimated during the orbit-determination process and that these accelerations continuously affect the state. For this reason, we map the covariance due to the process noise $\left(\mathbf{P}_{\omega}\right)$ only into the spacecraft velocity space using the matrix $\mathbf{B}$. In the actual computation the correlation time $\tau$ is set to 0.5 days and we vary the 
steady-state acceleration noise to study its impact on estimates of the gravity parameters. Between these measurements, the SRIF matrix is propagated by solving the above differential equations, and each measurement is incorporated into the SRIF matrix by

$$
\mathbf{R}\left(t_{k+1} ; t_{k+1}, t_{o}\right)=\mathbf{T}_{H}\left[\begin{array}{c}
\mathbf{R}\left(t_{k+1} ; t_{k}, t_{o}\right) \\
\boldsymbol{\Sigma}^{-1} \mathbf{H}_{t_{k+1}}
\end{array}\right]=\left[\begin{array}{c}
\mathbf{R}_{H}\left(t_{k+1} ; t_{k+1}, t_{o}\right) \\
0
\end{array}\right]
$$

The current-state covariance matrix is then obtained by computing,

$$
\mathbf{P}\left(t_{k+1} ; t_{k+1}, t_{o}\right)=\mathbf{R}_{H}^{-1}\left(t_{k+1} ; t_{k+1}, t_{o}\right) \mathbf{R}_{H}^{-T}\left(t_{k+1} ; t_{k+1}, t_{o}\right),
$$

which represents the a posteriori estimates of the state vector at $t=t_{k+1}$.

\section{Extracting Estimate Uncertainties}

Each component in a covariance matrix $\left(\mathbf{P}_{i j}=\sigma_{i j}\right)$ represents either the squares of standard deviation in the state or the correlation between two state variables. Hence, we can obtain the uncertainty estimates (i.e., standard deviations) of $\mathbf{p}$ by computing $\sigma_{i}=\sqrt{\sigma_{i i}}, i=n+1 \cdots n+m$. The unique opportunity of the heliocentric and Mercury-centric tests is the potential of separately estimating $\gamma, \beta$, and $J_{2}$; hence, it is of interest to analyze how these two parameters are correlated. We thus also find the correlation between $\gamma, \beta$, and $J_{2}$ by computing $\left(\sigma_{\gamma \beta} / \sqrt{\sigma_{\gamma \gamma} \sigma_{\beta \beta}}\right)=\left(\sigma_{\gamma \beta} / \sigma_{\gamma} \sigma_{\beta}\right)$, $\left(\sigma_{\gamma J 2} / \sqrt{\sigma_{\gamma \gamma} \sigma_{J 2 J 2}}\right)=\left(\sigma_{\gamma J 2} / \sigma_{\gamma} \sigma_{J 2}\right)$, and $\left(\sigma_{\beta J 2} / \sqrt{\sigma_{\beta \beta} \sigma_{J 2 J 2}}\right)=\left(\sigma_{\beta J 2} / \sigma_{\beta} \sigma_{J 2}\right)$, which can range between -1 and 1 . A value of zero represents a purely uncorrelated measurement whereas values close to 1 or -1 represent highly correlated or anti-correlated measurements.

\section{Measurement Data Types}

We only consider radiometric data types in this analysis and the possibility of implementing optical measurements is not considered. The first data type is two-way radar range measurements,

$$
Z_{R}=|\rho|=\rho,
$$

which measure the distance between the spacecraft and the tracking station based on the travel time of the up-link and down-link signals. Here, $\boldsymbol{\rho}$ is the vector from the Earth tracking station (Goldstone in our example) to the spacecraft.

The second data type we consider is Very Long Baseline Interferometry (VLBI) measurements, $Z_{V}$. VLBI measures the longitudinal and latitudinal angles of the spacecraft trajectory in the plane of sky of the tracking station [9]. Combined with range measurements, the 3-dimensional position of the spacecraft can be obtained. We represent this measurement as a set of angles,

$$
Z_{V}=\left[\begin{array}{ll}
Z_{m} & Z_{n}
\end{array}\right]^{T}
$$

where $Z_{m}$ and $Z_{n}$ are the longitudinal and the latitudinal angular measurements, respectively.

The final data type we consider are Doppler measurements, $Z_{D}$,

$$
Z_{D}=\hat{\boldsymbol{\rho}} \cdot \dot{\boldsymbol{\rho}}
$$

which are widely used for interplanetary missions. Here, $\hat{\boldsymbol{\rho}}$ is the unit vector of $\boldsymbol{\rho}$. This data type gives range-rate via Doppler frequency shifts in the transmitted signals and, due to the HamiltonMelbourne effect [10], provides angular information on the trajectory as well. 


\section{Error Sources}

\section{Station-Location Errors}

The only measurement parameter vector we consider is the station-location vector, which is defined as

$$
\mathbf{r}_{s l}=\left[\begin{array}{c}
R_{E} \sin \delta \\
R_{E} \cos \delta \\
\alpha
\end{array}\right]
$$

where $R_{E}$ is Earth mean radius $(6378 \mathrm{~km}), \alpha$ is the right ascension $\left(243.17^{\circ}\right)$, and $\delta$ is the declination $\left(54.67^{\circ}\right)$.

The only information needed are the partials of the observation vectors with respect to $\mathbf{r}_{s l}$. Taking partials of range measurements with respect to the station-location vector results in

$$
\frac{\partial Z_{R}}{\partial \mathbf{r}_{s l}}=-\hat{\boldsymbol{\rho}}^{T}\left(\frac{\partial \mathbf{r}_{t s}}{\partial \mathbf{r}_{s l}}\right)
$$

Taking partials of the VLBI measurements with respect to the station-location vector yields

$$
\left[\begin{array}{c}
\frac{\partial Z_{m}}{\partial \mathbf{r}_{s l}} \\
\frac{\partial Z_{n}}{\partial \mathbf{r}_{s l}}
\end{array}\right]=\left[\begin{array}{c}
-\frac{\hat{\mathbf{m}}_{o}^{T}}{\rho}\left(\frac{\partial \mathbf{r}_{t s}}{\partial \mathbf{r}_{s l}}\right) \\
-\frac{\hat{\mathbf{n}}_{o}^{T}}{\rho}\left(\frac{\partial \mathbf{r}_{t s}}{\partial \mathbf{r}_{s l}}\right)
\end{array}\right] .
$$

Finally, the partial derivatives of Doppler measurements are

$$
\frac{\partial Z_{D}}{\partial \mathbf{r}_{s l}}=-\frac{\dot{\boldsymbol{\rho}}^{T}}{\rho}\left(\mathbf{I}_{3 \times 3}-\hat{\boldsymbol{\rho}} \hat{\boldsymbol{\rho}}^{T}\right)\left(\frac{\partial \mathbf{r}_{t s}}{\partial \mathbf{r}_{s l}}\right)-\hat{\boldsymbol{\rho}}\left(\frac{\partial \mathbf{v}_{t s}}{\partial \mathbf{r}_{s l}}\right),
$$

where $\mathbf{v}_{t s}$ is the time derivative of $\mathbf{r}_{t s}$.

Uncertainties in the station-location corrupt the quality of radiometric measurements, and hence can significantly degrade the accuracy of our estimates of $\gamma, \beta$, and $J_{2}$. To analyze this effect, we assume that the uncertainty in the station-location vector is constant, and do not estimate $\mathbf{r}_{s l}$ in the covariance computation. Hence, this is in a sense a worst-case approach since we completely ignore the possibility of estimating $\mathbf{r}_{s l}$.

\section{Occultation Effects}

When the spacecraft passes in front of (or behind) the Sun or Mercury, we cannot obtain radiometric measurements. Since the trajectory originates close to the Sun, this can be an important effect in the experiments. For solar occultation, we assume no range measurements are taken when Sun-Earth-Probe (SEP) angle $\leq 5.27^{\circ}$ and no VLBI and Doppler measurements were taken when $\mathrm{SEP} \leq 0.77^{\circ}$. Also no radiometric measurements were taken when the Mercury-Earth-Probe angle $(\mathrm{MEP}) \leq 0.0016^{\circ}$

\section{Trajectory Models}

\section{Heliocentric Trajectory}

The spacecraft trajectory originating near the Sun is sensitive to GR. In this study, we consider the trajectory condition analyzed in Ref. [3], where the spacecraft is on a heliocentric hyperbolic trajectory (Figure 1) with perihelion located at four solar radii $r_{p}=4 R_{\odot}$ (where $R_{\odot}=$ solar radii $=$ $6.96 \times 10^{5} \mathrm{~km}$ ) with periapsis velocity $v_{p}=311 \mathrm{~km} / \mathrm{s}$. In this paper we will consider a larger range of possible orbit geometries, allowing for inclined orbits for the spacecraft. Let the state vector to be 


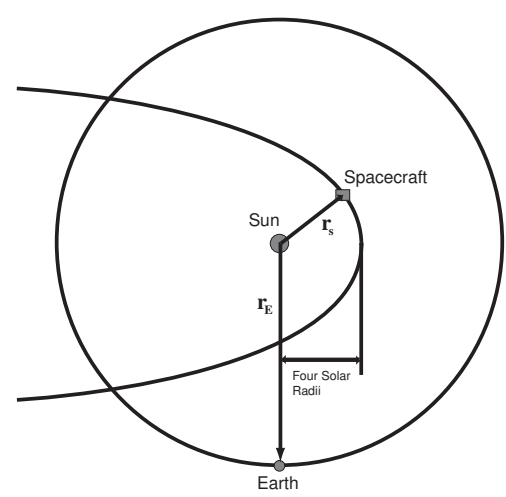

Figure 1: Heliocentric Spacecraft Trajectory.

estimated be $\mathbf{y}=\left[\begin{array}{lllll}\mathbf{r}_{s}^{T} & \mathbf{v}_{s}^{T} & \gamma & \beta & J_{2}\end{array}\right]^{T}$, where $\mathbf{r}_{s}$ and $\mathbf{v}_{s}$ are the spacecraft state vectors with the following acceleration model [11]:

$$
\begin{aligned}
\mathbf{a}_{s}= & \frac{\partial}{\partial \mathbf{r}_{M}}\left\{\frac{\mu_{\odot}}{r_{M}}\left[1+\frac{R_{\odot}^{2} J_{2}}{2 r_{M}^{4}}\left(r_{M}^{2}-3 z_{M}^{2}\right)\right]\right\} \\
& +\frac{\mu}{c^{2} r_{s}^{3}}\left[2(\gamma+\beta) \frac{\mu}{r_{s}} \mathbf{r}_{s}-\gamma v_{s}^{2} \mathbf{r}_{s}+2(\gamma+1)\left(\mathbf{r}_{s} \cdot \mathbf{v}_{s}\right) \mathbf{v}_{s}\right],
\end{aligned}
$$

where $r_{s}$ and $v_{s}$ are the magnitudes of $\mathbf{r}_{s}$ and $\mathbf{v}_{s}$, respectively, $\mu_{\odot}$ is the Sun's gravitational constant $\left(1.327 \times 10^{11} \mathrm{~km}^{3} / \mathrm{s}^{2}\right), c$ is the speed of light $\left(3 \times 10^{5} \mathrm{~km} / \mathrm{s}\right)$, and $J_{2}$ is the solar quadrupole moment $\left(J_{2}=2 \times 10^{-7}\right)[1$. In this case, we ignore the measurement parameters (i.e., station-location vector $\mathbf{r}_{s l}$ ) as discussed in Refs. [4, 5, 12 in great detail. We note that the GR perturbation only acts in the orbital plane, and therefore inclination and argument of ascending node are unchanged. The largest change in orbital elements due to $\gamma$ and $\beta$ occurs very early in the trajectory and essentially disappears after a few days. This is expected since the GR perturbation is at its maximum in close proximity to the Sun. One outcome of this observation is that it is highly desirable to extend radiometric tracking as close to the Sun as possible. Furthermore, Refs. 4, 12 discuss the importance of the partial derivatives of the orbital elements with respect to the GR parameters as the spacecraft travels along its trajectory. An important conclusion from these studies is that the partials of argument of periapsis, $\omega$, with respect to $\beta$ and $\gamma$ are distinct. The ratios of these partials, which represent the correlation between $\beta$ and $\gamma$, converge to a constant value more slowly than the ratios of the other orbital elements, indicating that there is sufficient information to disentangle $\gamma$ and $\beta$ by tracking the spacecraft close to perihelion.

The Earth's orbit is assumed to be circular with radius $r_{E}=1 \mathrm{AU}$ and we assume the initial Earth location to be along the Vernal Equinox. The spacecraft trajectory is obtained by numerically integrating the two-body equations of motion given in Eq. (25). Our hypothetical trajectory approaches perihelion on an elliptic orbit (with aphelion at Jupiter $\sim 5.2 \mathrm{AU}$ ), then boosts into a hyperbolic escape trajectory (using a perihelion maneuver of $\Delta V \sim 3 \mathrm{~km} / \mathrm{s}$ at $4 R_{\odot}$ ). Hence, to constrain the initial velocity error, we assume the presence of accelerometers to measure the large perihelion burn. At epoch the conservative initial uncertainties (variances) for the initial covariance matrix are given in Table 2 with zero correlations. The a priori uncertainties of $\gamma$ and $\beta$ are set at high values that assume no initial information. The a priori uncertainty of $J_{2}$ is an order of magnitude better than Mercury-centric case, this is because the heliocentric mission will likely to be carried out after the Mercury orbiter mission (i.e., such as the Solar probe mission).

Table 2: Initial Values of the Covariance Matrix (Heliocentric Case)

\begin{tabular}{ccccc}
\hline \hline$\sigma_{\mathbf{r}_{s}}(\mathrm{~km})$ & $\sigma_{\mathbf{v}_{s}}(\mathrm{~m} / \mathrm{s})$ & $\sigma_{\gamma}$ & $\sigma_{\beta}$ & $\sigma_{J 2}$ \\
\hline $\operatorname{diag}(1)$ & $\operatorname{diag}(1)$ & 1 & 1 & $10^{-8}$ \\
\hline \hline
\end{tabular}


We consider all three types of radiometric data types for this case and the resulting measurement partials are as follows:

$$
\begin{gathered}
\mathbf{h}_{R}=\left[\begin{array}{cc}
\hat{\boldsymbol{\rho}}^{T} & 0_{1 \times 5}
\end{array}\right], \\
\mathbf{h}_{V}=\left[\begin{array}{cc}
\frac{\hat{\mathbf{m}}_{o}^{T}}{\rho} & 0_{1 \times 5} \\
\frac{\hat{\mathbf{n}}_{o}^{T}}{\rho} & 0_{1 \times 5}
\end{array}\right], \\
\mathbf{h}_{D}=\left[\begin{array}{lll}
\dot{\boldsymbol{\rho}}^{T}\left(\frac{\partial \hat{\boldsymbol{\rho}}}{\partial \mathbf{r}_{s}}\right)^{T} & \hat{\boldsymbol{\rho}}^{T} & 0_{1 \times 2}
\end{array}\right],
\end{gathered}
$$

where we define

$$
\begin{aligned}
\hat{\mathbf{l}}_{o} & =\hat{\boldsymbol{\rho}} \\
\hat{\mathbf{m}}_{o} & =\hat{\mathbf{l}}_{o} \times \hat{\mathbf{n}}_{o}, \\
\hat{\mathbf{n}}_{o} & =\frac{\hat{\mathbf{z}}-\left(\hat{\mathbf{z}} \cdot \hat{\mathbf{l}}_{o}\right) \hat{\mathbf{l}}_{o}}{\left|\hat{\mathbf{z}}-\left(\hat{\mathbf{z}} \cdot \hat{\mathbf{l}}_{o}\right) \hat{\mathbf{l}}_{o}\right|} \\
\frac{\partial \hat{\boldsymbol{\rho}}}{\partial \mathbf{r}_{s}} & =\frac{1}{\rho}\left(\mathbf{I}_{3 \times 3}-\hat{\boldsymbol{\rho}} \hat{\boldsymbol{\rho}}^{T}\right),
\end{aligned}
$$

$\hat{\mathbf{z}}=\left[\begin{array}{lll}0 & 0 & 1\end{array}\right]^{T}$ and $\hat{\boldsymbol{\rho}}$ is the unit vector of $\boldsymbol{\rho}$. The measurements partial matrix and measurement noise matrix are then

$$
\begin{gathered}
\mathbf{H}=\left[\begin{array}{c}
\mathbf{h}_{R} \\
\mathbf{h}_{V} \\
\mathbf{h}_{D}
\end{array}\right], \\
\boldsymbol{\Sigma}=\left[\begin{array}{ccc}
\sigma_{R} & 0 & 0 \\
0 & \sigma_{V} & 0 \\
0 & 0 & \sigma_{D}
\end{array}\right] .
\end{gathered}
$$

For more discussion, the reader is referred to Refs. 4, 5, 12 ,

\section{Mercury-centric Trajectory}

In this case we track the Mercury-centric orbiter to measure Mercury's state and extract information on the Sun's gravity field. Figure 2 shows a schematic of the Earth, Mercury, and spacecraft orientations. Unlike the heliocentric case, both the transient and secular effect of GR become of importance in estimating the gravity parameters. The changes in Mercury's orbital elements due to GR are shown in Figure 3 [13. The changes in the semi-major axis $\Delta a$ and eccentricity $\Delta e$ oscillate over time, but the changes in argument of perihelion $\Delta \omega$ and mean anomaly $\Delta M$ have secular deviations from the mean value in addition to their transient oscillations. An important advantage of using Mercury's orbit is that the stochastic perturbations acting on it are small. The spacecraft itself is under the influence of stochastic perturbations (on the order of $\sigma_{a}=10^{-12} \mathrm{~km} / \mathrm{s}^{2}$ ). While non-gravitational perturbations (due to solar radiation, reflected solar radiation, planet thermal radiation, and solar wind, and other effects discussed by Longuski et al. [14]) are relatively large on a typical spacecraft, these perturbations are negligible on the planet because its area-to-mass ratio is vanishingly small as compared to a spacecraft. It is the large area-to-mass ratio of a spacecraft that makes the vehicle susceptible to non-gravitational forces. Ideally, we would like to put a transponder on the surface of Mercury to directly and precisely measure the planet's state. We assume that Mercury's equations of motion are governed by the PPN metric and the spacecraft equations of motions are governed solely by Mercury's gravity field (Newtonian). Figure 3 shows the geometry of the Earth, Mercury, and the spacecraft. Let the state to be estimated be denoted 


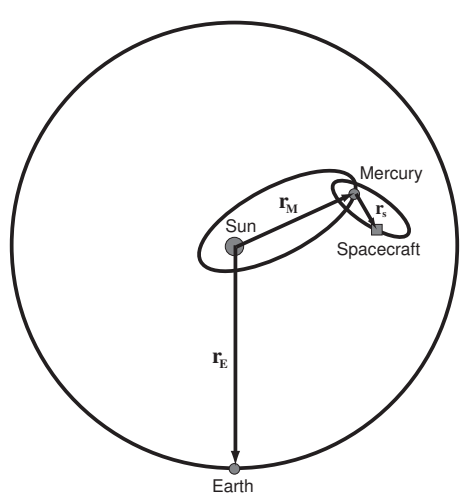

Figure 2: Mercury-centric Spacecraft Trajectory.

as $\mathbf{y}=\left[\begin{array}{llllllll}\mathbf{r}_{M}^{T} & \mathbf{v}_{M}^{T} & \mathbf{r}_{s}^{T} & \mathbf{v}_{s}^{T} & \gamma & \beta & J_{2} & \mathbf{r}_{s l}^{T}\end{array}\right]^{T}$, where the dynamic variables are $\mathbf{r}_{M}, \mathbf{v}_{M}, \mathbf{r}_{s}$, and $\mathbf{v}_{s}$, the parameters are $\gamma, \beta$, and $J_{2}$, and the measurement parameters are $\mathbf{r}_{s l}$. Here, $\mathbf{r}_{M}$ and $\mathbf{v}_{M}$ are Mercury's state vectors and $\mathbf{r}_{s}$ and $\mathbf{v}_{s}$ are the spacecraft state vectors. The accelerations of Mercury and the spacecraft are given as follows:

$$
\begin{aligned}
\mathbf{a}_{M}= & \frac{\partial}{\partial \mathbf{r}_{M}}\left\{\frac{\mu_{\odot}}{r_{M}}\left[1+\frac{R_{\odot}^{2} J_{2}}{2 r_{M}^{4}}\left(r_{M}^{2}-3 z_{M}^{2}\right)\right]\right\} \\
& +\frac{\mu_{\odot}}{c^{2} r_{M}^{3}}\left[2(\gamma+\beta) \frac{\mu_{\odot}}{r_{M}} \mathbf{r}_{M}-\gamma v_{M}^{2} \mathbf{r}_{M}+2(\gamma+1)\left(\mathbf{r}_{M} \cdot \mathbf{v}_{M}\right) \mathbf{v}_{M}\right], \\
\mathbf{a}_{s}= & -\frac{\mu_{M}}{r_{s}^{3}} \mathbf{r}_{s},
\end{aligned}
$$

where $\mu_{M}$ is Mercury's gravitational parameter $\left(\mu_{M}=22030 \mathrm{~km}^{3} / \mathrm{s}^{2}\right)$. The initial conditions for Mercury and the spacecraft are assumed to be at perihelion with orbital elements given in Table 3.

Table 3: Initial Orbital Elements of the Mercury and Spacecraft

\begin{tabular}{ccccccc}
\hline \hline & $a_{o}$ & $e_{o}$ & $i_{o}$ & $\omega_{o}$ & $\Omega_{o}$ & $M$ \\
\hline Mercury $^{\mathrm{a}}$ & $0.387 \mathrm{AU}$ & 0.206 & $7^{\circ}$ & $77^{\circ}$ & $48^{\circ}$ & $0^{\circ}$ \\
Spacecraft $^{\mathrm{b}}$ & $10136.2 \mathrm{~km}$ & 0.740 & $80^{\circ}$ & $60^{\circ}$ & $0^{\circ}$ & $0^{\circ}$ \\
\hline \hline
\end{tabular}

a. Orbital elements with respect to the Sun.

b. Orbital elements with respect to the Mercury.

The initial values of the covariance matrix are given in Table 4 with zero initial correlation, where we assume recent estimates of the parameters $\gamma$ and $J_{2}$. Also assumed are station-location accuracies on
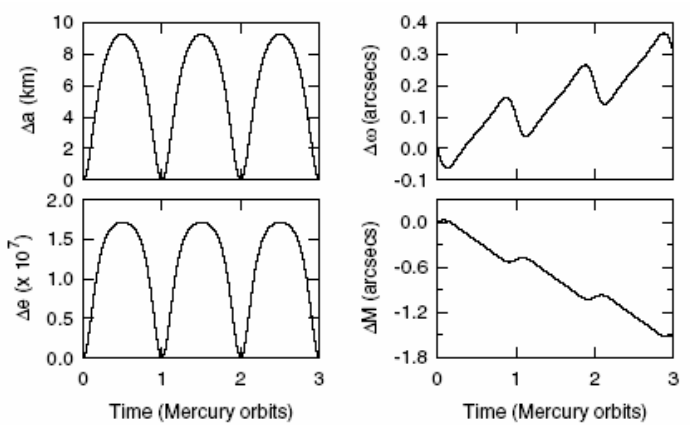

Figure 3: Changes in Mercury's orbital elements due to the effect of GR. 
the order of $1 \mathrm{~cm}$. We note that the station-location vector is not estimated in this analysis; however, its effect is included when radiometric measurements are updated. We assume more accurate initial uncertainties on the spacecraft state since the spacecraft state must be known accurately for capture at Mercury.

Table 4: Initial Values of the Covariance Matrix (Mercury-centric Case)

\begin{tabular}{ccccccc}
\hline \hline$\sigma_{\mathbf{r}_{M}}(\mathrm{~km})$ & $\sigma_{\mathbf{v}_{M}}(\mathrm{~m} / \mathrm{s})$ & $\sigma_{\mathbf{r}_{s}}(\mathrm{~km})$ & $\sigma_{\mathbf{v}_{s}}(\mathrm{~m} / \mathrm{s})$ & $\sigma_{\gamma}$ & $\sigma_{\beta}$ & $\sigma_{J 2}$ \\
\hline $\operatorname{diag}(0.1)$ & $\operatorname{diag}(0.1)$ & $\operatorname{diag}(0.1)$ & $\operatorname{diag}(0.1)$ & $10^{-4}$ & $10^{-1}$ & $10^{-7}$ \\
\hline \hline
\end{tabular}

The linear mapping matrix $\mathbf{A}$ is given as

$$
\begin{aligned}
\mathbf{A}(t) & =\frac{\partial \dot{\mathbf{y}}}{\partial \mathbf{y}}, \\
\mathbf{A}(t)= & {\left[\begin{array}{cccc}
\frac{\partial \dot{\mathbf{x}}_{M}}{\partial \mathbf{x}_{M}} & \frac{\partial \dot{\mathbf{x}}_{M}}{\partial \mathbf{v}_{M}} & \frac{\partial \dot{\mathbf{x}}_{M}}{\partial\left(\gamma, \beta, J_{2}\right)^{T}} & \frac{\partial \dot{\mathbf{x}}_{M}}{\partial \mathbf{r}_{s l}} \\
\frac{\partial \dot{\mathbf{x}}_{s}}{\partial \mathbf{x}_{M}} & \frac{\partial \dot{\mathbf{x}}_{s}}{\partial \mathbf{v}_{M}} & \frac{\partial \dot{\mathbf{x}}_{s}}{\partial\left(\gamma, \beta, J_{2}\right)^{T}} & \frac{\partial \dot{\mathbf{x}}_{s}}{\partial \mathbf{r}_{s l}} \\
\frac{\partial\left(\dot{\gamma}, \dot{\beta}, \dot{J}_{2}\right)^{T}}{\partial \mathbf{x}_{M}} & \frac{\partial\left(\dot{\gamma}, \dot{\beta}, \dot{J}_{2}\right)^{T}}{\partial \mathbf{v}_{M}} & \frac{\partial\left(\dot{\gamma}, \dot{\beta}, \dot{J}_{2}\right)^{T}}{\partial\left(\gamma, \beta, J_{2}\right)^{T}} & \frac{\partial\left(\dot{\gamma}, \dot{\beta}, \dot{J}_{2}\right)^{T}}{\partial \mathbf{r}_{s l}} \\
\frac{\partial \dot{\mathbf{r}}_{s l}}{\partial \mathbf{x}_{M}} & \frac{\partial \dot{\mathbf{x}}_{s l}}{\partial \mathbf{v}_{M}} & \frac{\partial \dot{\mathbf{x}}_{s l}}{\partial\left(\gamma, \beta, J_{2}\right)^{T}} & \frac{\partial \dot{\mathbf{x}}_{s l}}{\partial \mathbf{r}_{s l}}
\end{array}\right] . }
\end{aligned}
$$

We propagate the SRIF matrix including the stochastic acceleration perturbations acting on the velocity of the spacecraft (i.e., current state analysis, Eq. 14). In this case, we only consider the range and Doppler measurements with following measurement partials:

$$
\begin{aligned}
& \mathbf{h}_{R}=\left[\begin{array}{lllll}
\hat{\boldsymbol{\rho}}^{T} & \mathbf{0}_{1 \times 3} & \hat{\boldsymbol{\rho}}^{T} & \mathbf{0}_{1 \times 6} & \frac{\partial Z_{R}}{\partial \mathbf{r}_{s l}}
\end{array}\right],
\end{aligned}
$$

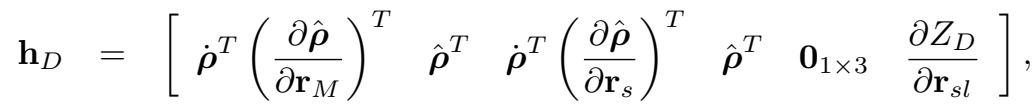

where

$$
\frac{\partial \hat{\boldsymbol{\rho}}}{\partial \mathbf{r}_{M}}=\frac{\partial \hat{\boldsymbol{\rho}}}{\partial \mathbf{r}_{s}}=\frac{1}{\rho}\left(\mathbf{I}_{3 \times 3}-\hat{\boldsymbol{\rho}} \hat{\boldsymbol{\rho}}^{T}\right)
$$

Now the measurement partial matrix $\mathbf{H}$ can be stated as

$$
\mathbf{H}=\left[\begin{array}{l}
\mathbf{h}_{R} \\
\mathbf{h}_{D}
\end{array}\right]
$$

with the measurement noise matrix

$$
\boldsymbol{\Sigma}=\left[\begin{array}{cc}
\sigma_{R} & 0 \\
0 & \sigma_{D}
\end{array}\right]
$$

\section{Results}

\section{Heliocentric Trajectory}

Assuming no error sources are present, unbiased estimates of $\sigma_{\gamma}=8.90 \times 10^{-5}$ and $\sigma_{\beta}=$ $4.09 \times 10^{-4}$ are achievable from the heliocentric trajectory 5. Figure 4 shows uncertainties in $\gamma$ and $\beta$ (ignoring effect of $J_{2}$ ) and their correlations as functions of the phase angle $\phi$ (i.e., initial Earth-Sun-probe angle). In this example, a zero degree inclination is considered and the estimates are taken at the end of 10-day timespan with all three measurement capabilities. It shows that 

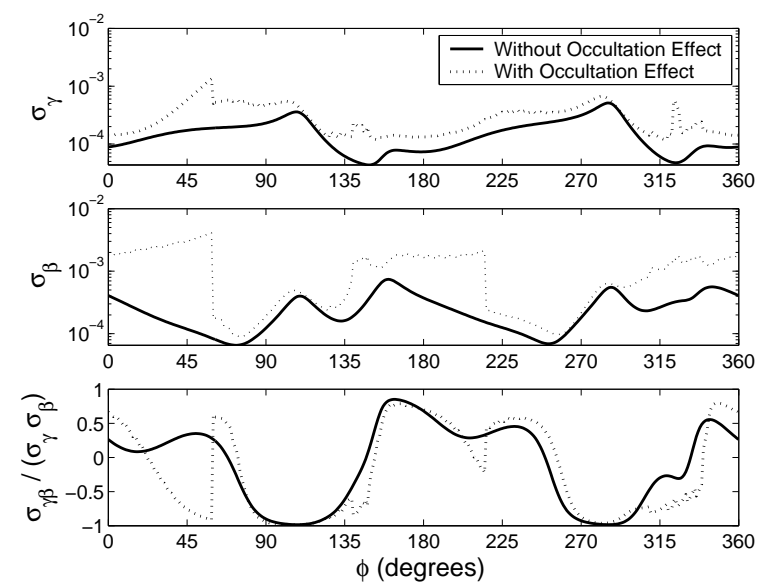

Figure 4: Heliocentric mission: accuracies of the parameter $\gamma$ and $\beta$ and their correlations for $i=0^{\circ}$ as functions of the phase angle $\phi$.

the solar occultation becomes an important error source, which requires careful trajectory design considerations to minimize this effect.

For this phase of our analysis, we repeated runs for a series of different inclinations, to determine how this changes the results. We expect this to have an influence, as it becomes possible to minimize solar occultations for some geometries. A highly inclined orbit can be achieved using a Jupiter flyby. For the following examples, we assume no error sources are included and present the effect of the spacecraft trajectory orientations on the estimates of $\gamma, \beta$, and $J_{2}$ using X-band tracking capability. Figures 5-10 show uncertainties of $\gamma, \beta$, and $J_{2}$ as functions of $\omega$ and $\Omega$ for inclinations of $45^{\circ}$ and $90^{\circ}$. All the estimates are taken at the end of a 10-day timespan with 15-minute measurement update. Table 5 summarizes the best (minimum) and worst (maximum) estimates of these gravity parameters with corresponding orbit elements $\omega$ and $\Omega$. The estimates of $\gamma, \beta$, and $J_{2}$ vary 1-3 orders of magnitude by changing orientation of the spacecraft orbit.

Table 5: Best and Worst Estimates of $\gamma, \beta$, and $J_{2}$ for $i=45^{\circ}$ and $i=90^{\circ}$.

\begin{tabular}{c|ccc|ccc}
\hline \hline $\begin{array}{c}i \\
(\mathrm{deg})\end{array}$ & $\begin{array}{c}\text { Best } \\
\text { Estimate }\end{array}$ & $\begin{array}{c}\omega \\
(\mathrm{deg})\end{array}$ & $\begin{array}{c}\Omega \\
(\mathrm{deg})\end{array}$ & $\begin{array}{c}\text { Worst } \\
\text { Estimate }\end{array}$ & $\begin{array}{c}\omega \\
(\mathrm{deg})\end{array}$ & $\begin{array}{c}\Omega \\
(\mathrm{deg})\end{array}$ \\
\hline 45 & $\sigma_{\gamma}=6.59 \times 10^{-5}$ & 35 & 5 & $\sigma_{\gamma}=3.00 \times 10^{-3}$ & 330 & 105 \\
90 & $\sigma_{\gamma}=1.04 \times 10^{-4}$ & 225 & 5 & $\sigma_{\gamma}=7.40 \times 10^{-3}$ & 75 & 85 \\
45 & $\sigma_{\beta}=1.27 \times 10^{-4}$ & 280 & 0 & $\sigma_{\beta}=4.06 \times 10^{-3}$ & 150 & 285 \\
90 & $\sigma_{\beta}=8.42 \times 10^{-5}$ & 300 & 0 & $\sigma_{\beta}=1.11 \times 10^{-2}$ & 195 & 270 \\
45 & $\sigma_{J 2}=7.65 \times 10^{-9}$ & 250 & 0 & $\sigma_{J 2}=5.11 \times 10^{-8}$ & 330 & 105 \\
90 & $\sigma_{J 2}=3.21 \times 10^{-9}$ & 250 & 355 & $\sigma_{J 2}=1.95 \times 10^{-7}$ & 10 & 90 \\
\hline \hline
\end{tabular}

One important result to note is that $\sigma_{\gamma}, \sigma_{\beta}$, and $\sigma_{J_{2}}$ are highly sensitive to spacecraft orbit orientations, analogous to the sensitivity of $\sigma_{\gamma}$ and $\sigma_{\beta}$ to the initial phase angle ( $\phi$ for the planar problem (i.e., $i=0^{\circ}$, Figure 4) [4, 5, 12. When solar occultation effects are included, the range of uncertainties change, but the basic trend of the uncertainty distributions remains the same. For $i=90^{\circ}$ the estimates become highly degraded near $\Omega= \pm 90^{\circ}$. One clear explanation comes from the fact that the spacecraft orbit is perpendicular to the Earth line-of-sight, decreasing the information content of both range and Doppler. Moreover, there are regions where $i=45^{\circ}$ case gives better estimates of $\gamma, \beta$, and $J_{2}$ than $i=90^{\circ}$ case. This indicates that the spacecraft trajectory must be designed optimally in order to carry out this mission. 


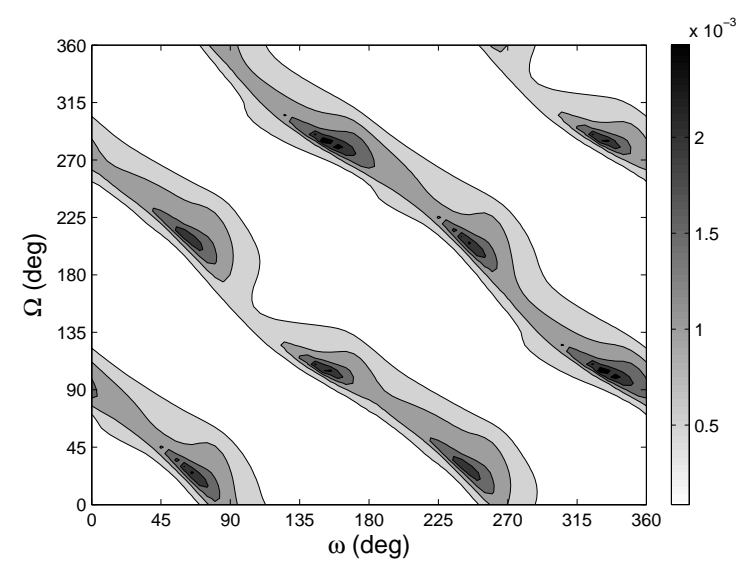

Figure 5: Heliocentric mission: accuracies of the parameter $\gamma$ for $i=45^{\circ}$; the white areas correspond to accuracies on the order of $7 \times 10^{-5}$.

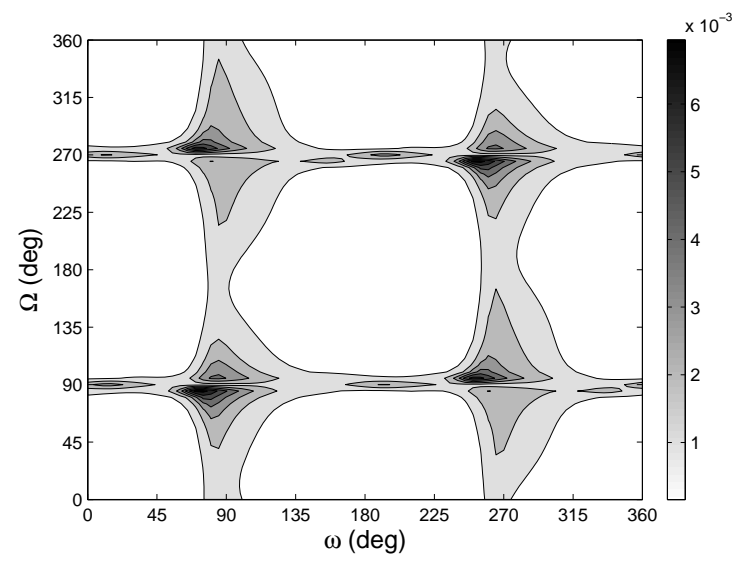

Figure 6: Heliocentric mission: accuracies of the parameter $\gamma$ for $i=90^{\circ}$; the white areas correspond to accuracies on the order of $2 \times 10^{-4}$.

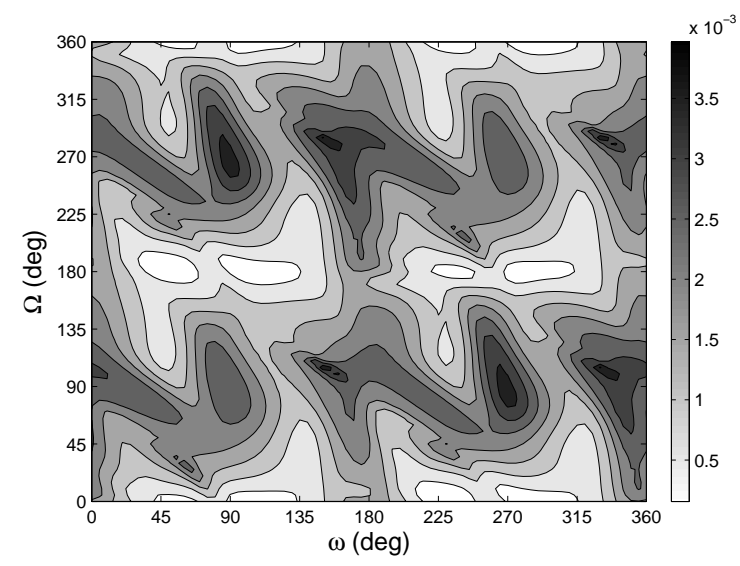

Figure 7: Heliocentric mission: accuracies of the parameter $\beta$ for $i=45^{\circ}$; the white areas correspond to accuracies on the order of $2 \times 10^{-4}$. 


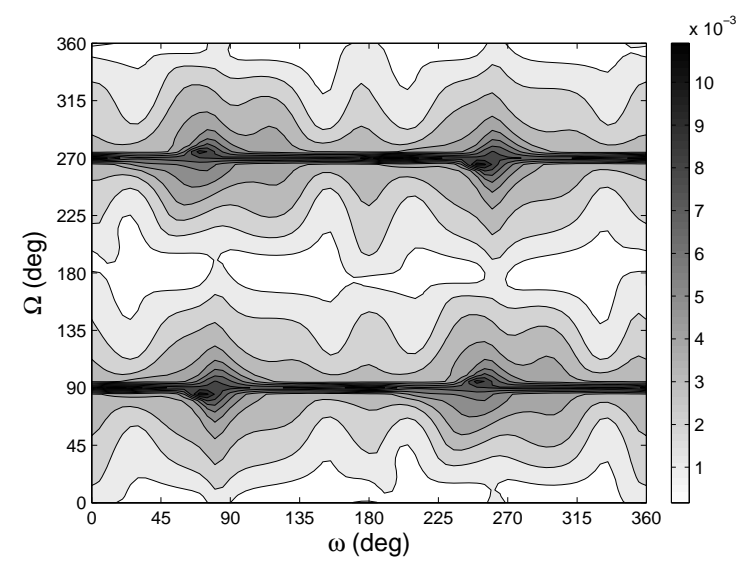

Figure 8: Heliocentric mission: accuracies of the parameter $\beta$ for $i=90^{\circ}$; the white areas correspond to accuracies on the order of $9 \times 10^{-5}$.

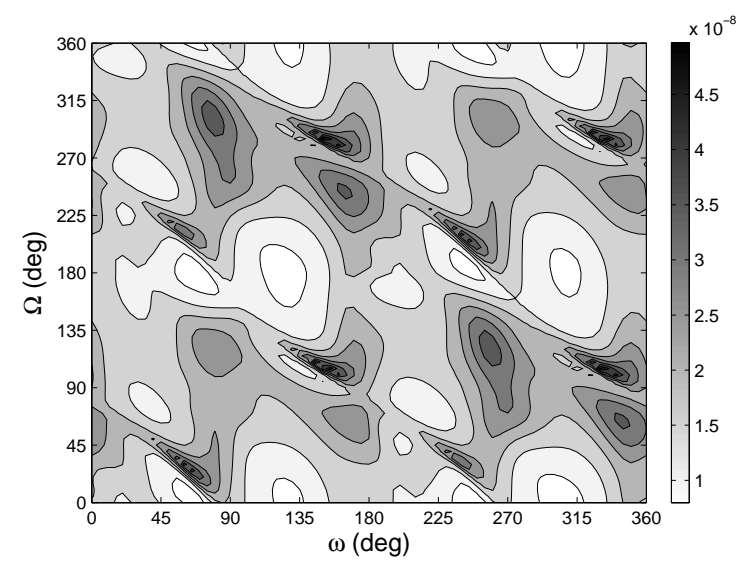

Figure 9: Heliocentric mission: accuracies of the parameter $J_{2}$ for $i=45^{\circ}$; the white areas correspond to accuracies on the order of $8 \times 10^{-9}$.

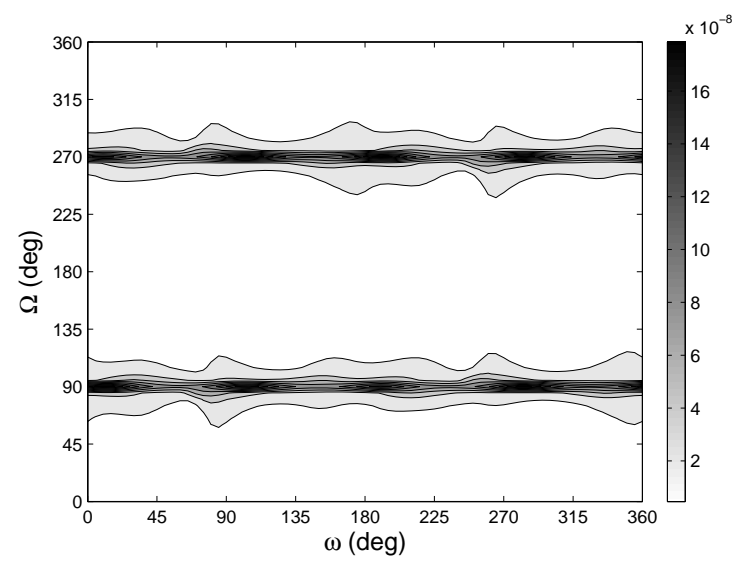

Figure 10: Heliocentric mission: accuracies of the parameter $J_{2}$ for $i=90^{\circ}$; the white areas correspond to accuracies on the order of $4 \times 10^{-9}$. 


\section{Mercury-centric Trajectory}

The simulation of the Mercury orbiter was carried out for a period of 100 days with 15-minute measurement updates assuming X-band capability for both range and Doppler data. Figures 1113 show the evolution of the uncertainties in Mercury's position $\left(\sigma_{r_{M}}=\sqrt{\sigma_{x}^{2}+\sigma_{y}^{2}+\sigma_{z}^{2}}\right)$ and the gravity parameters $\left(\sigma_{\gamma}, \sigma_{\beta}, J_{2}\right)$ as functions of time for $\sigma_{a}=10^{-10} \mathrm{~km} / \mathrm{s}^{2}$. These uncertainties $(\sigma$ 's) represent the 1- $\sigma$ level standard deviations. The result shows that we will not be able to obtain meaningful estimates of the gravity parameters for such a large value of stochastic accelerations (i.e., $\left.\sigma_{a}=10^{-10} \mathrm{~km} / \mathrm{s}^{2}\right)$. However, when we consider $\sigma_{a}=10^{-12} \mathrm{~km} / \mathrm{s}^{2}$ as shown in Figures 14-16, the parameter $\beta$ can be measured to a significant accuracy (even if we only consider the Doppler measurements). For the current interplanetary missions, $\sigma_{a}$ is assumed to be on the order of $10^{-12} \mathrm{~km} / \mathrm{s}^{2}$ [14. These levels of uncertainties on the gravity parameters can also be reduced if we consider a longer timespan. As expected, the correlations between all three constants are very high and further analysis is needed to disentangle gravity information from these radiometric measurements. Table 6 summarizes the estimates of $\gamma, \beta$, and $J_{2}$ by showing the a posteriori taken at the end of a 100-day timespan. These results are directly related to the MESSENGER mission, which was launched on August 3, 2004 and will arrive at Mercury in 2011 after series of flybys. The steady-state stochastic acceleration for the MESSENGER mission is assumed to be between $10^{-12}$ $\mathrm{km} / \mathrm{s}^{2}$ and $10^{-10} \mathrm{~km} / \mathrm{s}^{2}$ and is equipped with the X-band tracking capability. The most challenging problem in this study will be to minimize the uncertainty in the solar radiation pressure, which is the largest non-gravitational force acting on the spacecraft. We note that the Bepi-Colombo mission (future ESA mission to Mercury) has $\sigma_{a}$ on the order of $10^{-12} \mathrm{~km} / \mathrm{s}^{2}$ and has the K-band tracking capability, which can provide improved estimates of the gravity parameters $\gamma, \beta$, and $J_{2}$.

Table 6: Estimates of $\gamma, \beta$, and $J_{2}$ for $\sigma_{a}=10^{-10} \mathrm{~km} / \mathrm{s}^{2}$ and $\sigma_{a}=10^{-12} \mathrm{~km} / \mathrm{s}^{2}$ taken at the end of a 100-day timespan.

\begin{tabular}{clrccc}
\hline \hline$\sigma_{a}\left(\mathrm{~km} / \mathrm{s}^{2}\right)$ & Measurement Type & $\sigma_{r_{M}}(\mathrm{~m})$ & $\sigma_{\gamma} \times 10^{5}$ & $\sigma_{\beta} \times 10^{3}$ & $\sigma_{J_{2}} \times 10^{8}$ \\
\hline $10^{-10}$ & Range Only & 15.96 & 9.88 & 1.72 & 9.43 \\
$10^{-10}$ & Doppler Only & 329.82 & 9.99 & 5.37 & 9.89 \\
$10^{-10}$ & Range and Doppler & 8.66 & 9.59 & 1.44 & 8.43 \\
$10^{-12}$ & Range Only & 3.18 & 8.90 & 0.83 & 6.02 \\
$10^{-12}$ & Doppler Only & 10.40 & 9.72 & 1.55 & 9.04 \\
$10^{-12}$ & Range and Doppler & 0.71 & 6.90 & 0.33 & 3.76 \\
\hline \hline
\end{tabular}

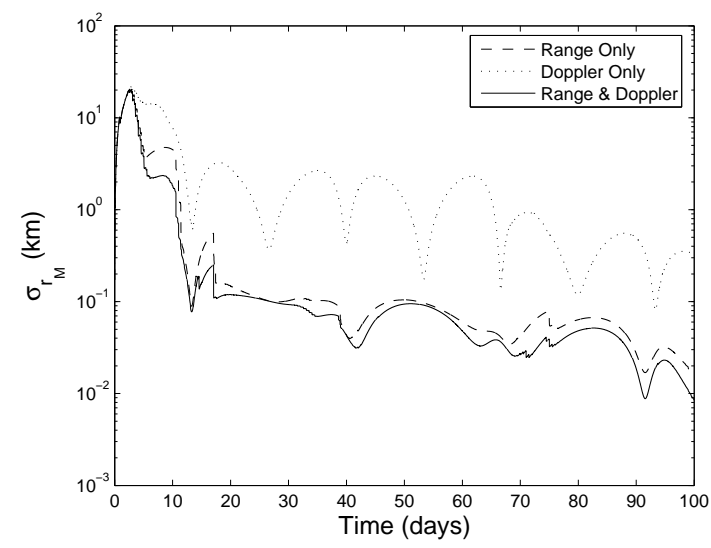

Figure 11: Mercury orbiter mission: accuracy of Mercury's Position for $\sigma_{a}=10^{-10} \mathrm{~km} / \mathrm{s}^{2}$. 

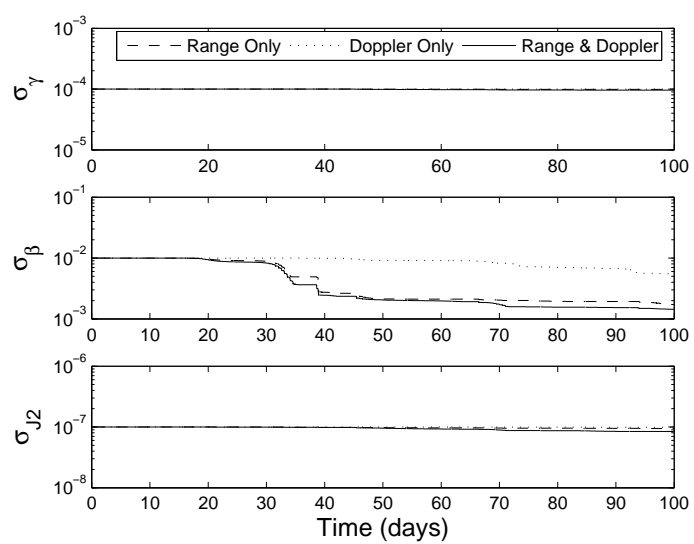

Figure 12: Mercury orbiter mission: accuracies of the gravity parameters $\gamma, \beta$, and $J_{2}$ for $\sigma_{a}=10^{-10}$ $\mathrm{km} / \mathrm{s}^{2}$.
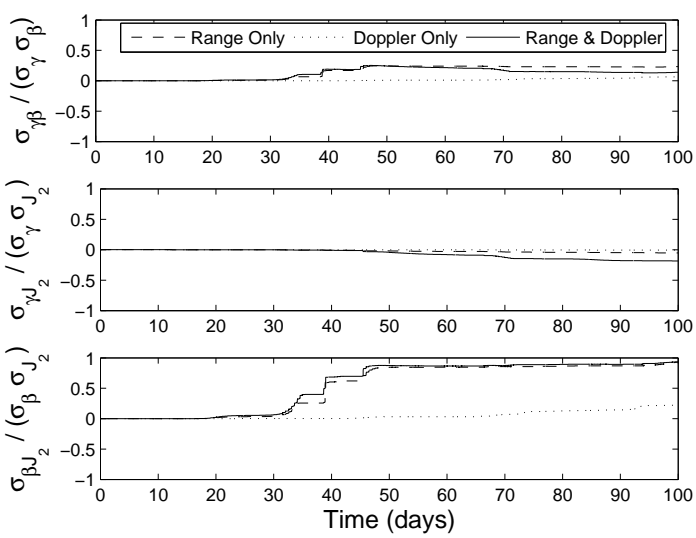

Figure 13: Mercury orbiter mission: correlations of the gravity parameters $\gamma, \beta$, and $J_{2}$ for $\sigma_{a}=$ $10^{-10} \mathrm{~km} / \mathrm{s}^{2}$.

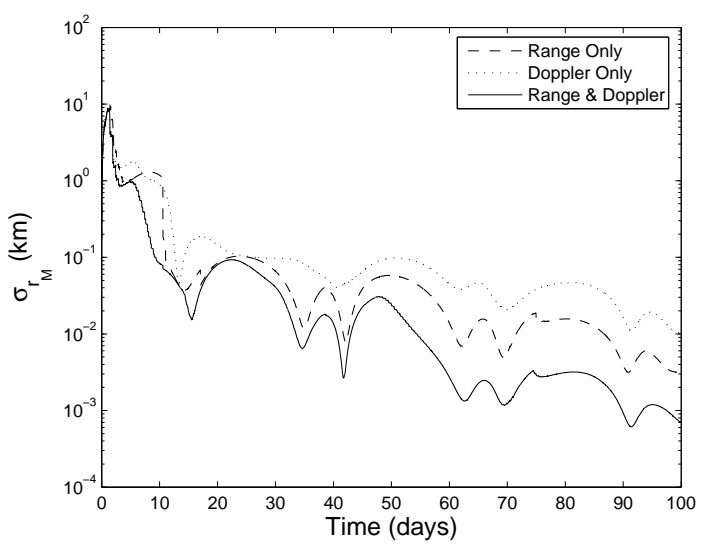

Figure 14: Mercury orbiter mission: accuracy of Mercury's Position for $\sigma_{a}=10^{-12} \mathrm{~km} / \mathrm{s}^{2}$. 

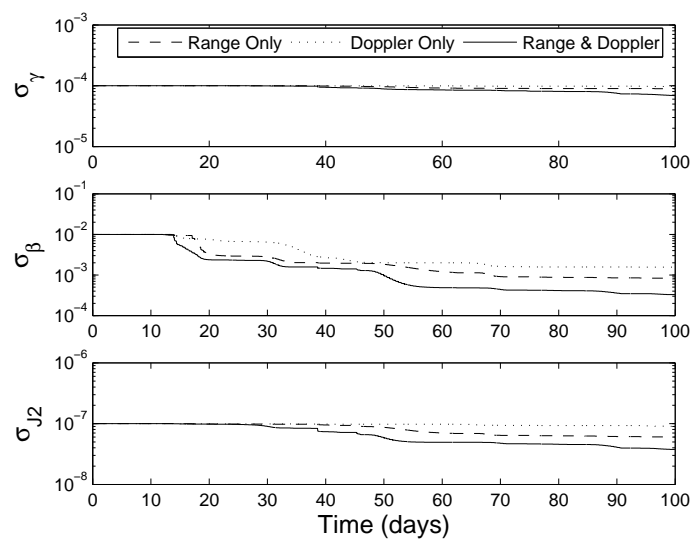

Figure 15: Mercury orbiter mission: accuracies of the gravity parameters $\gamma, \beta$, and $J_{2}$ for $\sigma_{a}=10^{-12}$ $\mathrm{km} / \mathrm{s}^{2}$.
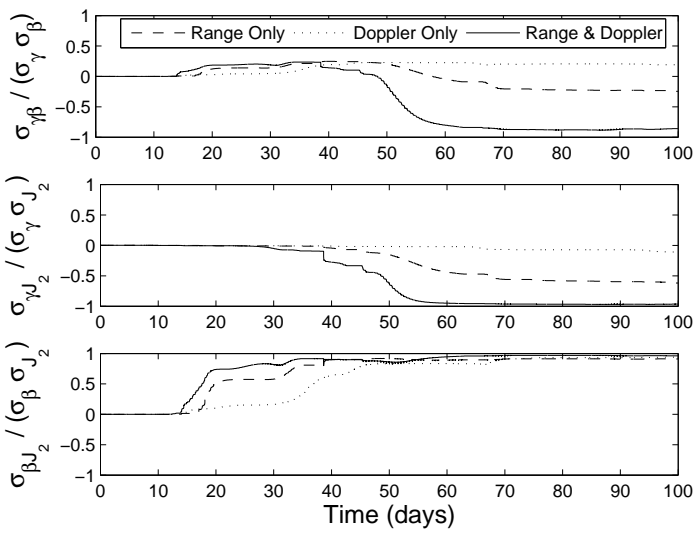

Figure 16: Mercury orbiter mission: correlations of the gravity parameters $\gamma, \beta$, and $J_{2}$ for $\sigma_{a}=$ $10^{-12} \mathrm{~km} / \mathrm{s}^{2}$.

\section{Conclusions}

In this paper, we have analyzed how well the PPN parameters and solar quadrupole moment can be estimated from spacecraft radiometric tracking data such as range, VLBI, and Doppler measurements.

In the heliocentric mission, the spacecraft originates from its perihelion $\left(r_{p}=4 R_{\odot}\right)$ with $v_{p}=311$ $\mathrm{km} / \mathrm{s}$ and we obtain radiometric measurements as it propagates along the heliocentric trajectory. The uncertainties in $\gamma, \beta$, and $J_{2}$ are estimated using the initial state covariance analysis and resulting accuracies of these parameters for $i=45^{\circ}$ and $i=90^{\circ}$ are presented. As it was shown in Table 5 , the uncertainties in the gravity parameters vary 1-3 orders of magnitude by changing the orbit orientations, and hence, the spacecraft trajectory must be carefully designed in order to ensure significant estimates.

Also discussed is the possibility of estimating these gravity parameters by tracking a Mercury orbiter. Gravity information is extracted from the range and Doppler radiometric measurements while the spacecraft orbits Mercury over a long timespan. The results show that the parameters $\gamma$, $\beta$, and $J_{2}$ can be estimated to a significant level. An important item to note is that the parameters $\beta$ and $J_{2}$ have never been measured directly. One usually assumes GR as a valid theory of gravitation and measures $J_{2}$; or measures the PPN parameters based on a given solar model. Hence, this indicates that the MESSENGER mission to Mercury can provide significant measurements of both GR and the Sun.

We have demonstrated, under ideal conditions, the feasibility of carrying out GR experiments with currently available technology. For the heliocentric case, the spacecraft must be equipped 
with either drag-free technology or accurate accelerometers, and the trajectory must be designed optimally in order to minimize the parameter uncertainties. The challenge for the Mercury orbiter case is to reduce the stochastic perturbations acting on the spacecraft as much as possible.

\section{Acknowledgments}

The research described in this paper was sponsored by the Interplanetary Network Directorate Technology Program at the Jet Propulsion Laboratory, California Institute of Technology which is under contract with the National Aeronautics and Space Administration. The first author would like to thank John D. Anderson and Sami W. Asmar from Radio Science group at Jet Propulsion Laboratory, California Institute of Technology for supporting this research and their helpful comments.

\section{References}

[1] Pireaux, S. and Rozelot, J.-P., "Solar Quadrupole Moment and Purely Relativistic Gravitation Contributions to Mercury's Perihelion Advance," Astrophysics and Space Science, Vol. 284, 2003, pp. 1159 - 1194 .

[2] Bertotti, B., Iess, L., and Tortora, P., "A Test of General Relativity using Radio Links with the Cassini Spacecraft," Nature, Vol. 425, 2003, pp. $374-376$.

[3] Longuski, J. M., Fischbach, E., and Scheeres, D. J., "Deflection of Spacecraft Trajectories as a New Test of General Relativity," Phys. Rev. Lett., Vol. 86, No. 14, 2001, pp. $2942-2945$.

[4] Longuski, J. M., Fischbach, E., Scheeres, D. J., Giampieri, G., and Park, R. S., "Deflection of Spacecraft Trajectories as a New Test of General Relativity: Determining the PPN Parameters $\beta$ and $\gamma, "$ Phys. Rev. D, Vol. 69, No. 042001, 2004, pp. $042001-1-042001-15$.

[5] Park, R. S., Scheeres, D. J., Giampieri, G., Longuski, J. M., and Fischbach, E., "A Test of General Relativity: Estimating the Parameterized Post-Newtonian Parameters from Spacecraft Radiometric Measurements," Accepted for publication in Journal of Spacecraft and Rockets, 2004.

[6] Bierman, G. J., Factorization Methods for Discrete Sequential Estimation, Academic Press, 1977, pp. 69-76.

[7] Montenbruck, O. and Gill, E., Satellite Orbits, Springer, 2nd Edition, 2001, pp. 257-291.

[8] Scheeres, D. J., Han, D., and Hou, Y., "Influence of Unstable Manifolds on Orbit Uncertainty," Journal of Guidance, Control, and Dynamics, Vol. 24, 2001, pp. $573-585$.

[9] Thurman, S. W. and Sybert, C. B., "Planetary Approach Orbit Determination Using EarthBased Short and Long Baseline Radio Interferometry," Advances in the Astronautical Sciences, Vol. 76, Pt. 2, 1992, pp. 1039 - 1053.

[10] Hamilton, T. W. and Melbourne, W. G., "Information Content of a Single Pass of Doppler Data from a Distant Spacecraft," JPL Space Programs Summary, Vol. 3, 1966, pp. 18 - 23.

[11] Will, C. M., Theory and Experiment in Gravitational Physics, Cambridge University Press, Cambridge, UK, 1993, sec. 7.

[12] Park, R .S., Scheeres, D. J., Giampieri, G., Longuski, J. M., and Fischbach, E., "Estimating General Relativity Parameters from Radiometric Tracking of Heliocentric Trajectories," Feb. 2003, 13th AIAA/AAS Space Flight Mechanics Conference, Ponce, Puerto Rico.

[13] Balogh, A. and Giampieri, G., "Mercury: the planet and its orbit," Rep. Prog. Phys., Vol. 65, 2002, pp. $529-560$.

[14] Longuski, J. M., Todd, R. E., and Koenig, W. W., "Survey of Nongravitational Forces and Space Environmental Torques: Applied to the Galileo," Journal of Guidance, Control, and Dynamics, Vol. 15, No. 3, 1992, pp. $545-553$. 